\title{
Damage repair
}

Bacteria are present in large numbers in the intestines of many animals, but how the host maintains the integrity of its tissues in the presence of these bacteria is still poorly understood. In a recent issue of Genes \& Development,

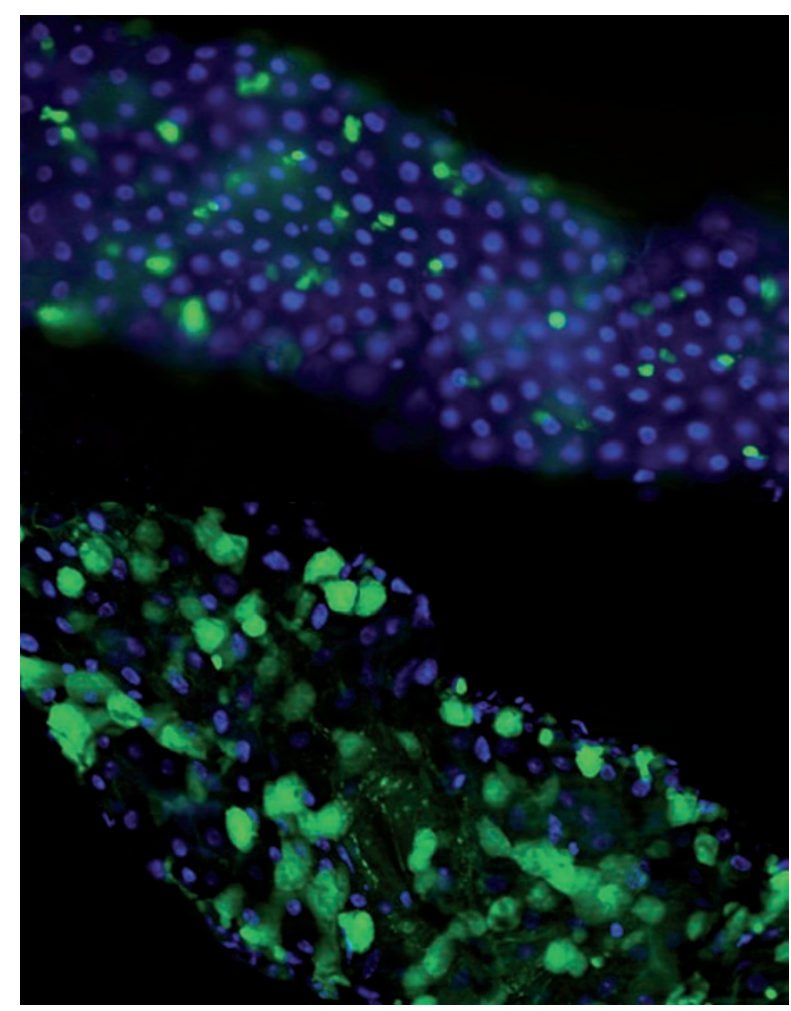

Ingestion of the Gram-negative bacterium Erwinia carotovora subsp. carotovora str. 15 by Drosophila melanogaster provokes a massive increase in epithelial renewal through increased intestinal stem cell proliferation and differentiation (the top panel shows uninfected flies and the bottom panel shows infected flies), as revealed by the expression of an Escargot-green fluorescent protein reporter. Nuclei are stained with 4',6-diamidino-2-phenylindole (DAPI; in blue). Image courtesy of N. Buchon, Ecole Polytechnique Fédérale de Lausanne, Switzerland.
Buchon and colleagues show that repair of the intestinal epithelium in Drosophila melanogaster after challenge with foreign bacteria requires an oxidative burst and multiple signalling pathways.

An oxidative burst that is regulated by the NADPH oxidase dual oxidase (Duox) is part of the immune response in the gut of $D$. melanogaster. This damages the intestine wall, which requires epithelial renewal through proliferation of intestinal stem cells (ISCs). Buchon and colleagues found that antioxidants decreased ISC proliferation in D. melanogaster in response to challenge with the non-commensal bacterium Erwinia carotovora subsp. carotovora str. 15 (Ecc15). Inhibition of Duox also decreased ISC proliferation in the presence of $E c c 15$, indicating that the oxidative burst plays an important part in initiating the proliferative repair of gut tissues.

In D. melanogaster that are challenged with $E c c 15$, transcription is upregulated for members of the Janus kinase-signal transducer and activator of transcription (JAK-STAT) pathway, which helps to regulate the proliferation of stem cells in D. melanogaster, and the Jun N-terminal kinase (JNK) pathway, which is involved in the regulation of tissue integrity in the guts of older flies. The authors found that the inhibition of signalling through either pathway prevented ISC proliferation in the presence of $E c c 15$. Furthermore, they found that one of the proteins that is upregulated by the JAK-STAT pathway, unpaired 3 (Upd3), has an important role in signalling to the stem cells to induce proliferation.

Although Ecc15 does not kill wild-type flies, flies that lacked the proliferative response died 4 to 8 days after challenge. When the flies were challenged with a low dose of a pathogen, a proliferative response like that of wild-type flies was detected, but high doses led to epithelial damage and mortality, indicating that the repair pathway is insufficient in certain conditions. When the authors compared conventionally reared flies and axenic flies, which do not have bacteria in their intestines, they found that signalling through the JNK and JAK-STAT pathways and cell proliferation were decreased in the axenic flies.

It therefore seems that tissue morphology and the response to oxidative damage in the gut are maintained by recognition of the oxidative burst and signalling through the JAK-STAT and JNK pathways, leading to ISC proliferation and repair.

Christiaan van Ooij

ORIGINAL RESEARCH PAPER Buchon, N., Broderick, N.A., Chakrabarti, S. \& Lemaitre, B. Invasive and indigenous microbiota impact intestinal stem cell activity through multiple pathways in Drosophila. Genes Dev. 23. 2333-2344 (2009) 